\title{
Ready for the next generation of monitoring biosensors? L.M.Lechuga
}

Laura M. Lechuga is a full professor of the Spanish National Research Council and the Head of the Nanobiosensors and Bioanalytical Applications Group at the Catalan Institute of Nanoscience and Nanotechnology in Barcelona (Spain). Her research focuses on the development of nanophotonic biosensors, their integration on lab-on-a-chip platforms, and their application for environmental and clinical diagnostics. She has published more than 250 articles, has 8 families of patents, and has delivered more than 320 invited talks. She has co-founded two spin-off companies and has received several awards and distinctions.

The main responsibility for the steady degradation of our environment is the human being activity. But the environment has turned the tables, and the profound interlink between environmental pollution and human health status is more evident every day. Air and water pollution are two examples of contamination producing acute and chronic effects, and even death, on humans.

Monitoring the environment contamination and its implication in the malfunctioning of the human health is a must needed endeavor. Environmental monitoring has experienced a significant progress in the last decades and, nowadays, we account with extremely accurate and sensitive analytical techniques. But these methods are limited to centralized laboratories, require expensive instrumentation, are time-consuming, and are in need of trained personnel. On-site and real-time evaluation of contaminants is vital to manage environmental degradation and to protect the human health of the consequences, but this evaluation is an elusive problem.

Biosensor devices are promising environmental monitoring tools because they are specific, sensitive, fast, and reusable. A biosensor is a device that uses specific biological receptors to detect chemical and biological compounds usually by electrical, mechanical, or optical signals. The continuous progress in biosensor technologies is offering outstanding analytical tools for portable and on-site environmental monitoring, thanks to their capability of miniaturization, multiplexing, label-free detection, and integration in labon-a-chip platforms. This special issue covers the most relevant biosensor technologies which have been proposed for on-site environmental surveillance, showing how these novel sensing technologies are valuable alternative solutions for the existing environmental monitoring tools currently used.

In this special issue, Marquez and Narvaez explain one of the most impactful sensor technology born in this century as it is paper-based biosensors, highlighting in their article the challenges and opportunities of this new branch of sensing devices. This low-cost sensing technology is aimed to solve real-world demands as it is environmental and health diagnosis and their interlink. The review explains how, given its versatility, paper can be exploited as a microfluidic network, preconcentration platform and/or microextraction membrane accompanied by specialized sensing zones. Furthermore, paper-based analytical devices intended for environmental monitoring can be supported by ubiquitous gadgets such as smartphones and sample collection drones.

Hoon Kim et al. analyze how the environmental monitoring can be performed using aptamer-based biosensors. This type of biosensors has received special attention because of the unique advantages which aptamers can offer, as they can specifically recognize diverse target molecules (from small molecules to bacteria). Aptamers constitute a viable alternative to conventional biological receptor as they usually are more stable than, for example, antibodies. This contribution describes the most recent updates on the application of aptasensors in the detection of contaminants in real samples, including water and soil samples, with very promising results. In addition, the main challenges still to be addressed for the practical and commercial applications of biosensors based on aptamers are critically discussed in the manuscript. 
Bryan and Miller describe in their contribution, a type of powerful biosensors based on the optical properties of silicon. They mainly focussed on two classes of silicon-based biosensors capable of multiplex detection: sensors using free-space optics, relying on changes in the reflective properties of the substrate on target molecule binding, and integrated photonic sensors, relying on refractive index variations. Both technologies exhibit a great potential for environmental monitoring, opening the door to a multiplexed analysis of different analytes using the same sample volume, as authors explain in their manuscript.

Zinkl and Wegener focus on cell-based biosensors as valuable tools to screen for the impact of environmental pollutants on human health. Cell-based assays can be monitored by non-invasive, label-free readout using electrochemical impedance sensors. This review mainly summarizes the state-of-the-art of this powerful biosensor technology, which allows for a time-resolved observation of various phenotypic assays related to changes in cell spreading, migration, proliferation or differentiation, which can be affected by the contaminants. In addition, this technology has a strong potential for performing assays sequentially, in such a way that several phenotypes can be accessible from only one exposure campaign.

Cardenosa-Rubio et al. focus in one specific branch of optical evanescent wave biosensors, the microring optical resonators, which have emerged as an exceptional choice in biosensing because of their sensitivity, small footprint, and multiplex configuration, fabrication with standard low-cost technologies, besides their capacity to operate under a real-time and label-free detection scheme. The review explains the most recent and relevant applications achieved in environmental analysis and in clinical diagnosis for this promising and highly compact technology.

Finally, Díaz-González and Fernandez-Sanchez report on one of the most used biosensors, the electrochemical ones, as one of the best candidates for providing deployable tools for on-site analysis. These biosensors can be used for the detection of priority pollutants, and moreover, they can be engaged as a surveillance alert system. This review provides an excellent overview on the most recent advances in the fabrication of such compact electroanalytical biosensors with the potential for a rapid translation to decentralized studies of water contamination events. 